\title{
COMPETENCIA PARA EL CUIDADO EN EL HOGAR Y CARGA EN CUIDADORES FAMILIARES DE ADULTOS Y NIÑOS CON CÁNCER
}

\author{
Gloria Mabel Carrillo ${ }^{1}$, Sonia Patricia Carreño², Laura Milena Sánchez ${ }^{3}$
}

\section{Resumen}

Objetivo. Describir y comparar la competencia para el cuidado en el hogar y la carga de los cuidadores familiares de niños con cáncer, con la de cuidadores familiares de adultos con cáncer. Método. Estudio cuantitativo, exploratorio y comparativo con 160 cuidadores familiares de personas con cáncer en tratamiento activo en dos centros de atención oncológica en Bogotá, Colombia (2016), quienes una vez caracterizados respondieron el instrumento de competencia para el cuidado en el hogar (CUIDAR) y la escala de sobrecarga para el cuidado. Resultados. Las características de los cuidadores familiares de niños y adultos con cáncer guardan semejanzas en género, ocupación, nivel educativo y apoyos con los que se cuenta. Los cuidadores de niños experimentan mayor carga para el cuidado en comparación con los de adultos con cáncer, y tienen una competencia mayor para el cuidado en el hogar y en las dimensiones conocimiento, disfrutar y anticipación. Conclusiones. Se requiere focalizar aún más el apoyo a cuidadores familiares, según el receptor de cuidado. Programas para fortalecer la competencia de cuidado en el hogar y disminuir la carga son necesarios para atender y responder a la necesidad de los cuidadores familiares de personas con cáncer. Financiación. Facultad de Enfermería. Universidad Nacional de Colombia.

\footnotetext{
1 Autor principal. Enfermera. Magíster en Enfermería, Doctora en Enfermería. Profesora Asociada, Universidad Nacional de Colombia, Sede Bogotá, Facultad de Enfermería, Departamento de Enfermería. gmcarrillog@unal.edu.co. Carrera 30 No. 45-01 Edificio 228 Oficina 302. Bogotá-Colombia. (005713165000 ext. 17011) Móvil 573144704609

${ }^{2}$ Enfermera, Magíster en Enfermería, Doctora en Enfermería. Profesora Auxiliar. Universidad Nacional de Colombia, Sede Bogotá, Facultad de Enfermería. Departamento de Enfermería. spcarrenom@unal.edu.co

${ }^{3}$ Enfermera, Magíster en Enfermería, Universidad Nacional de Colombia, Sede Bogotá, Facultad de Enfermería. Departamento de Enfermería. laumisanchez@hotmail.com
} 


\title{
HOME CARE COMPETENCY AND BURDEN IN FAMILY CAREGIVERS OF ADULTS AND CHILDREN WITH CANCER
}

\author{
Gloria Mabel Carrillo, Sonia Patricia Carreño, Laura Milena Sánchez
}

\section{Abstract}

Objective. To describe and compare the home care competency and the burden of family caregivers of children with cancer, with that of family caregivers of adults with cancer. Method. Quantitative, exploratory and comparative study with 160 family caregivers of people with cancer in active treatment in two oncology care centers in Bogotá -Colombia (2016), who once characterized answered the competence instrument for home care (CUIDAR) and the care burden scale. Results. The characteristics of family caregivers of children and adults with cancer have similarities in gender, occupation, educational level and available assistance. Child caregivers experience a greater care burden in comparison with those of adults with cancer, and have a greater competence for home care and in the dimensions of knowledge, enjoyment and anticipation. Conclusions. It is necessary to focus even more the support to family caregivers, according to the receiver of care. Programs to strengthen home care competency and reduce the burden are necessary to meet and respond to the needs of family caregivers of people with cancer. Financing. Nursing School. National university of Colombia. 


\section{COMPETÊNCIA PARA CUIDAR EM CASA E CARGA EM FAMILIARES CUIDADORES DE ADULTOS E CRIANÇAS COM CÂNCER}

Lilia Suarez Puerto, Javier Díaz Castro, José William Hernández González

\section{Resumo}

Objetivo. Descrever e comparar a competência para cuidar em casa e a carga dos cuidadores familiares de crianças com câncer, com aquela dos cuidadores familiares de adultos com câncer. Método. Estudo quantitativo, exploratório e comparativo com 160 cuidadores familiares de pessoas com câncer em tratamento ativo em dois centros de atenção oncológica em Bogotá, Colômbia (2016), que uma vez caracterizadas responderam o instrumento de competência para cuidados em casa (CUIDAR) e a escala de sobrecarga para atendimento. Resultados. As características dos cuidadores familiares de crianças e adultos com câncer apresentam semelhanças em gênero, ocupação, nível educacional e suporte disponível. Os cuidadores de crianças vivenciam maior carga de cuidado quando comparados aos cuidadores de adultos com câncer, e possuem maior competência para o cuidado em casa e nas dimensões conhecimento, desfrutar e antecipação. Conclusões. É necessário focar ainda mais o apoio aos familiares cuidadores, de acordo com o receptor de cuidados. Programas para fortalecer a competência do cuidado em casa e reduzir a carga são necessários para atender e responder às necessidades dos cuidadores familiares de pessoas com câncer. Financiamento. Escola de Enfermagem. Universidade Nacional de Colômbia. 


\section{Introducción}

El cáncer es una enfermedad crónica que ha aumentado considerablemente, como resultado del incremento en la esperanza de vida de la población y la exposición a factores de riesgo modificables, entre otros aspectos (1). Se presenta en todos los grupos etarios y representa una de las mayores causas de muerte en el mundo, causando un alto impacto económico y requiriendo tratamientos largos y de alta complejidad. Con frecuencia ocasiona dependencia física, psicológica y social, reduce el estado funcional, generando la necesidad de contar con un cuidador familiar que asuma un rol activo en el proceso de atención.

La inclusión del cuidador familiar aparece como un elemento central y a la vez, un reto para la práctica clínica de oncología que implica caracterizarlo, definir y evaluar sus competencias para el cuidado en el hogar, así como el diseño de las estrategias de seguimiento y acompañamiento que corresponde a las instituciones que prestan los servicios de salud.

El tratamiento oncológico, pese a ofrecer un beneficio para lograr la curación o evitar el progreso de la enfermedad, acarrea riesgos durante su administración y posterior a la misma que deben ser conocidos por la persona con cáncer y su cuidador en el domicilio. Se debe garantizar la continuidad del tratamiento en casa que fue iniciado a nivel intrahospitalario, en tal sentido la planeación del egreso permite reducir la estancia hospitalaria, las tasas de reingreso y la morbilidad, y atención eficaz con disminución de costos (2).
Los cuidadores de personas con cáncer han de adquirir habilidades relacionadas con el conocimiento en la administración de medicamentos, control de los síntomas y soportes en actividades básicas diarias, así como los posibles efectos adversos que se han de presentar.

Sin embargo, estas nuevas responsabilidades pueden generar carga en el cuidador familiar y la competencia para el cuidado en el hogar se consolida como una variable moduladora de dicha carga (3).

Abordar la competencia para el cuidado en el hogar y la carga de estos cuidadores se constituye como una temática de relevancia clínica y a la vez, de alto interés investigativo. El conocimiento que se tenga sobre el grado de complejidad de la situación crónica, la competencia del cuidador principal para ofrecer cuidados y los apoyos disponibles para el cuidado permiten establecer prioridades de acción (4).

Los cuidadores primarios de niños con cáncer asumen mayores responsabilidades; son usualmente madres, que presentan alto riesgo para generar algunos problemas como la depresión, desórdenes de estrés postraumático, estrés, dificultades económicas y conflictos familiares con consecuencias sobre su calidad de vida (5).

La amplia gama de responsabilidades que debe asumir el cuidador primario de un niño con cáncer requiere el desarrollo de un nivel óptimo de competencias necesarias para cuidarlo en el hogar para que con ello mejore los resultados en el receptor de cuidados y en sí mismo, inmerso en ello, la prevención o mitiga- 
ción de la sobrecarga del cuidador. Se ha documentado que la competencia para cuidar en el hogar en los cuidadores primarios de niños con cáncer se encuentra en niveles medios a bajos, mientras que la sobrecarga del cuidador es ausente (6).

La competencia para cuidar en el hogar en este contexto es entendida como la capacidad, habilidad y preparación que tiene el cuidador familiar de la persona con cáncer para ejercer la labor de cuidar y que comprende el bienestar, como la satisfacción personal con la calidad de vida percibida; las condiciones personales para cuidar, como aquellas características de la personalidad que son base para que se afronte el rol, los problemas y desafíos; la interacción y relación social, entendidos como el uso y satisfacción con las relaciones sociales y redes de apoyo que fortalecen el cuidado; el conocimiento que comprende las nociones e ideas respecto a la enfermedad, terapéuticas, manejo de dispositivos y cuidados en las actividades de la vida diaria; la anticipación, que se refiere a la capacidad de predecir las necesidades de la persona cuidada, y lo instrumental, que se refiere a las habilidades y destrezas para realizar las labores de cuidado directo (6).

Estudios previos con cuidadores familiares de adultos con cáncer en quimioterapia (7), con cuidadores de personas en falla cardíaca (8) y con cuidadores de pacientes con otras enfermedades crónicas $(8,9,10)$ reportan niveles medios de competencia en general y por dimensiones, siendo muy bajo el porcentaje con niveles óptimos esperados.

Aunque se ha documentado la vivencia de los cuidadores de adultos o niños con cáncer, se han reconocido necesidades de cuidado y se han diseñado modelos para abordarlas, aún no se encuentra en la literatura, reportes que comparen las competencias de los cuidadores de adultos con los cuidadores de niños. Una comparación entre la competencia para el cuidado en el hogar de cuidadores familiares de niños y adultos con cáncer es necesaria para explorar y desarrollar estrategias de capacitación y acompañamiento con apoyos más focalizados, frente a la competencia de cuidado específica de cada grupo, abriendo nuevos caminos para dar respuestas desde la experiencia a los los cuidadores de personas con cáncer en la transición hospital-hogar.

El objetivo del estudio fue comparar la competencia y la carga percibida para el cuidado en el hogar de cuidadores familiares de niños con cáncer frente a la de los cuidadores de adultos con cáncer.

\section{Materiales y Métodos}

Estudio de abordaje cuantitativo, corte transversal realizado en el 2016 con 160 cuidadores familiares personas con cáncer, organizados en dos grupos: uno de 80 cuidadores familiares de adultos con diagnóstico de cáncer y tratamiento activo y otro de 80 cuidadores de niños con diagnóstico y tratamiento activo por cáncer. Se trató de un muestreo por conveniencia en el que los cuidadores familiares de personas con cáncer, quienes eran atendidos en las unidades de oncología, estaban invitados a participar del estudio. De ese modo, llenaban los formularios, dirigidos por las investigadoras. La aplicación individual de los formularios 
tuvo una duración entre 35 y 55 minutos. Los sujetos del estudio fueron cuidadores familiares adultos con vínculo de parentesco o cercanía con el adulto/ niño frente a quien cumplía las funciones de cuidado. Todos fueron captados a través de dos centros de referencia en oncología en Colombia.

\section{Instrumentos}

Competencia para el cuidado en el hogar (CUIDAR). Escala desarrollada en el contexto colombiano que consta de 60 ítems con graduación de tipo Likert calificada de 0 a 3 y se compone de 6 dimensiones: conocimiento (0-30 puntos), unicidad-condiciones personales (0-36 puntos), instrumental (024 puntos), disfrutar-bienestar (0-36 puntos), anticipación (0-18 puntos), relación e interacción social (0-36 puntos). Los puntajes más altos indican mayor grado de competencia. Las respuestas a los ítems se suman dando un puntaje total y uno para cada dimensión. Cuenta con pruebas psicométricas en español (11).

Percepción de carga del cuidado de Zarit: escala que logra medir multidimensionalmente el fenómeno de sobrecarga del cuidador, siendo la más usada a nivel internacional. Se utilizó la versión de 22 ítems en español, que incluye preguntas tipo Likert de 5 opciones (nunca, rara vez, algunas veces, bastantes veces y casi siempre). La puntuación va desde 22 a 110 puntos y la sobrecarga se califica: ausencia de sobrecarga (< o igual a 46), sobrecarga ligera (47 a 55), sobrecarga intensa ( $>$ o igual a 56 ). Cuenta con pruebas psicométricas en español (12) y para Colombia (13).
Encuesta de caracterización para el cuidado de la díada, conformada por la persona con enfermedad crónica y el cuidador familiar, que contiene tres dimensiones: condiciones y perfil sociodemográfico de la díada, percepción de carga y apoyos. La misma fue desarrollada y validada en el contexto de América Latina (10).

Dentro de las consideraciones éticas se tuvieron en cuenta las pautas CIOMS y los parámetros establecidos en la Resolución 8430 de 1993, expedida por el Ministerio de Salud de Colombia (14). Se aplicó el consentimiento informado y se obtuvo aval del Comité de Ética de la Universidad Nacional de Colombia, la Facultad de Enfermería, el Comité de Investigaciones del Instituto Nacional de Cancerología y una Institución de cuarto nivel en la Ciudad de Floridablanca, Santander. Fue considerada la norma actual de propiedad intelectual, en el marco de la cual se respetaron los derechos de autor en las fuentes primarias de literatura consultadas.

\section{Análisis}

Medias (desviación estándar) y medianas (valores mínimo, máximo) fueron calculadas para determinar la competencia y la carga del cuidador. La distribución de frecuencias y los porcentajes se utilizaron para presentar el perfil sociodemográfico.

La comparación de los grupos en la competencia para el cuidado y la carga del cuidador y en cada una de sus dimensiones se realizó a través de una prueba paramétrica para muestras pa- 
readas ( $\mathrm{t}$-student), previamente ratificados los supuestos de la prueba. El análisis estadístico se llevó a cabo, utilizando el paquete estadístico SPSS versión 23.0.

\section{Resultados}

\section{Características de los participantes}

En cuanto al perfil clínico, el tipo de cáncer que predomina en los niños es leucemia, seguido de tumores del sistema nervioso central y tumores de hueso; género masculino, edad promedio de 8,8 años con una desviación estándar (DS) de 4,92 .

En los adultos, en primer lugar se encuentran los linfomas, seguido de cáncer de mama, leucemias y mieloma múltiples, género femenino, edad promedio de 51,8 años con una DS de 16,96 (Tabla 1).

Tabla 1. Perfil clínico de los pacientes.

\begin{tabular}{|c|c|c|c|c|}
\hline VARIABLE & \multicolumn{2}{|c|}{$\begin{array}{c}\text { NIÑO CON CÁNCER } \\
n=80(\%)\end{array}$} & $\begin{array}{l}\text { ADULTO CON CÁNCER } \\
n=80(\%)\end{array}$ & CON \\
\hline \multirow[t]{7}{*}{ DIAGNÓSTICO } & Leucemia & 35 & Cáncer de ovario & 5 \\
\hline & Tumores del SNC & 20 & Cáncer gastrointestinal & 3,75 \\
\hline & Linfoma & 5 & Cáncer de mama & 23,75 \\
\hline & $\begin{array}{l}\text { Tumor en los } \\
\text { huesos }\end{array}$ & 17,5 & Mieloma múltiple & 12,5 \\
\hline & $\begin{array}{l}\text { Tumor celular } \\
\text { germinales }\end{array}$ & 3,75 & Linfoma & 25 \\
\hline & Retinoblastoma & 3,75 & Leucemia & 20 \\
\hline & Otros & 15 & Otros & 10 \\
\hline \multirow[t]{2}{*}{ GÉNERO } & Femenino & 46,25 & 63,75 & \\
\hline & Masculino & 53,75 & 36,25 & \\
\hline \multirow[t]{4}{*}{ EDAD } & Mínimo & 0,5 & 19 & \\
\hline & Máximo & 17 & 83 & \\
\hline & Media & 8,8 & 51,8 & \\
\hline & (DS) & 4,92 & 16,96 & \\
\hline
\end{tabular}

Fuente: Elaboración propia a partir de los datos de estudio, 2016. 


\section{Perfil sociodemográfico de los cuidadores}

Los cuidadores familiares de niños con cáncer tienen una edad promedio de 33,9 años con una DS de 7,3; la mayoría corresponde a madres que cuidan a sus hijos (87,5\%), únicas cuidadoras $(57,5 \%)$, de estratos socioeconómicos bajos $(66,25 \%)$, con predominancia de niveles educativos: primaria $(41,2 \%)$ y secundaria $(45 \%)$, con unión marital (63,75\%), ocupación hogar (68,75\%); con un promedio de 8,4 meses como cuidador (DS 9,9) y con una media de dedicación al cuidado de 22,3 horas al día (DS 4,4).
Los cuidadores familiares de adultos con cáncer tienen una edad promedio de 45,9 años con una DS de 14,7; la mayoría mujeres $(72,5 \%)$, con otros cuidadores de apoyo $(52,5 \%)$, de estratos socioeconómicos medios $(47,5 \%)$, con predominancia de niveles educativos secundaria $(41,25 \%)$ y primaria $(21,25 \%)$, con unión marital $(66,25 \%)$, ocupación hogar $(33,75 \%)$; con un promedio de 17,3 meses como cuidador (DS 22,6) y con una media de dedicación al cuidado de 15, 3 horas al día (DS 7,9) (Tabla 2).

Tabla 2. Caracterización del cuidador familiar.

\begin{tabular}{|c|c|c|c|}
\hline \multicolumn{2}{|c|}{ VARIABLE } & $\begin{array}{l}\text { Cuidador de niño } \\
\text { con cáncer } n=80 \\
(\%)\end{array}$ & $\begin{array}{l}\text { Cuidador de adulto } \\
\text { con cáncer } n=80(\%)\end{array}$ \\
\hline \multirow[t]{2}{*}{ Género } & Femenino & 87,5 & 72,5 \\
\hline & Masculino & 12,5 & 27,5 \\
\hline \multirow[t]{5}{*}{ Escolaridad } & Ninguna & 2,5 & 1,25 \\
\hline & Primaria & 41,25 & 21,25 \\
\hline & Secundaria & 45 & 41,25 \\
\hline & Técnico & 2,5 & 16,25 \\
\hline & Profesional & 8,75 & 20 \\
\hline \multirow[t]{2}{*}{ Estado marital } & $\mathrm{Si}$ & 63,75 & 66,25 \\
\hline & No & 36,25 & 33,75 \\
\hline \multirow[t]{5}{*}{ Ocupación } & Empleado & 12,5 & 22,5 \\
\hline & Estudiante & 3,75 & 6,25 \\
\hline & Hogar & 68,75 & 33,75 \\
\hline & Trabajo Independiente & 15 & 23,75 \\
\hline & Pensionado & 0 & 8,75 \\
\hline
\end{tabular}




\begin{tabular}{|c|c|c|c|}
\hline \multirow{3}{*}{$\begin{array}{l}\text { Estrato } \\
\text { socioeconómico }\end{array}$} & Bajo & 91,25 & 43,75 \\
\hline & Medio & 8,75 & 47,5 \\
\hline & Alto & 0 & 8,75 \\
\hline \multirow{2}{*}{$\begin{array}{l}\text { Cuida desde el } \\
\text { momento del } \\
\text { diagnóstico }\end{array}$} & $\mathrm{Si}$ & 100 & 96,25 \\
\hline & No & 0 & 3,75 \\
\hline \multirow[t]{2}{*}{ Único cuidador } & Sí & 57,5 & 47,5 \\
\hline & No & 42,5 & 52,5 \\
\hline \multirow[t]{4}{*}{ Edad en años } & Mínimo & 18 & 18 \\
\hline & Máximo & 51 & 79 \\
\hline & Media & 33,9 & 45,9 \\
\hline & DS & 7,3 & 14,7 \\
\hline \multirow{4}{*}{$\begin{array}{l}\text { Tiempo como } \\
\text { cuidador en meses }\end{array}$} & Mínimo & 1 & 3 \\
\hline & Máximo & 36 & 120 \\
\hline & Media & 8,4 & 17,3 \\
\hline & DS & 9,9 & 22,6 \\
\hline \multirow{4}{*}{$\begin{array}{l}\text { Horas diarias } \\
\text { dedicadas a cuidar }\end{array}$} & Mínimo & 6 & 3 \\
\hline & Máximo & 24 & 24 \\
\hline & Media & 22,3 & 15,3 \\
\hline & DS & 4,4 & 7,9 \\
\hline
\end{tabular}

Fuente: Elaboración propia, a partir de los datos de estudio, 2016.

Bienestar percibido y apoyos con los que cuentan

Tanto los cuidadores de niños como los de adultos con cáncer perciben altos niveles de apoyo familiar, religioso y so- cial. Los apoyos psicológico y económico son los más bajos.

Los cuidadores de adultos perciben más altos niveles de bienestar físico, psicológico, social y espiritual respecto a los cuidadores de niños (Tabla 3). 
INVESTIGACIONES ANDINA No. 36, Vol. 20

Tabla 3. Bienestar percibido y apoyo a cuidadores familiares.

\begin{tabular}{|c|c|c|c|c|}
\hline & VARIABLE & & $\begin{array}{c}\text { CUIDADOR DE } \\
\text { NIÑO CON CÁNCER } \\
\mathbf{n = 8 0}(\%)\end{array}$ & $\begin{array}{l}\text { CUIDADOR DE ADULTO } \\
\text { CON CÁNCER } n=80(\%)\end{array}$ \\
\hline \multirow{16}{*}{ 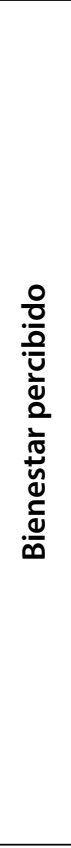 } & Físico & Bajo & 8,75 & 5 \\
\hline & & Medio & 20 & 12,5 \\
\hline & & Alto & 60 & 40 \\
\hline & & Muy alto & 11,25 & 42,5 \\
\hline & Psicológico & Bajo & 5 & 3,75 \\
\hline & & Medio & 47,5 & 18,75 \\
\hline & & Alto & 41,25 & 37,5 \\
\hline & & Muy alto & 6,25 & 40 \\
\hline & Social & Bajo & 1,25 & 3,75 \\
\hline & & Medio & 16,25 & 13,75 \\
\hline & & Alto & 66,25 & 45 \\
\hline & & Muy alto & 16,25 & 37,5 \\
\hline & Espiritual & Bajo & 1,25 & 0 \\
\hline & & Medio & 6,25 & 7,5 \\
\hline & & Alto & 58,75 & 31,25 \\
\hline & & Muy alto & 33,75 & 61,25 \\
\hline \multirow{15}{*}{ 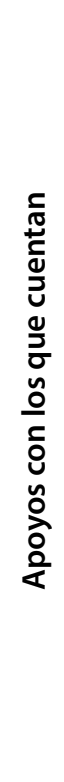 } & Psicológico & Bajo & 26,3 & 15,0 \\
\hline & & Medio & 27,5 & 15,0 \\
\hline & & Alto & 46,3 & 31,3 \\
\hline & Familiar & Bajo & 2,5 & 3,8 \\
\hline & & Medio & 26,3 & 3,8 \\
\hline & & Alto & 71,3 & 88,8 \\
\hline & Religioso & Bajo & 1,3 & 3,8 \\
\hline & & Medio & 13,8 & 8,8 \\
\hline & & Alto & 85,0 & 80,0 \\
\hline & Económico & Bajo & 13,8 & 7,5 \\
\hline & & Medio & 37,5 & 15,0 \\
\hline & & Alto & 48,8 & 70,0 \\
\hline & Social & Bajo & 3,8 & 6,3 \\
\hline & & Medio & 35,0 & 12,5 \\
\hline & & Alto & 61,3 & 61,3 \\
\hline
\end{tabular}

Fuente: Elaboración propia, a partir de los datos de estudio, 2016. 


\section{Sobrecarga del cuidador}

La sobrecarga para el cuidado es mayor en los cuidadores familiares de niños con cáncer respecto a la de los adultos, de forma global y por dimensiones. Al comparar, se identifica que hay una diferencia estadísticamente significativa $(\mathrm{p}<0.01)$ entre la sobrecarga de los cui- dadores de adultos y niños con cáncer, siendo la sobrecarga mayor en los cuidadores de niños. De igual manera, se observan diferencias $\mathrm{p}<0.01$ en las dimensiones impacto y competencias $y$ expectativas, siendo mayor la sobrecarga en los cuidadores de niños con cáncer. La dimensión interpersonal no tiene diferencias en los dos grupos (Tabla 4).

Tabla 4. Sobrecarga para el cuidador.

\begin{tabular}{lllllllll}
\hline \multirow{2}{*}{ Variable } & \multicolumn{2}{c}{$\begin{array}{c}\text { Cuidador niño con } \\
\text { cáncer }\end{array}$} & \multicolumn{2}{c}{$\begin{array}{c}\text { Cuidador adulto con } \\
\text { cáncer }\end{array}$} & t & & p \\
\cline { 2 - 5 } & Media & DS & Media & DS & & & \\
\hline Total Zarit & 52,1 & 15,9 & 38,1 & 11,0 & & 6,5 & $<0.01$ & \\
Impacto & 30,9 & 8,5 & 21,4 & 7,0 & & 7,6 & $<0.01$ & \\
Competencias y & 11,8 & 5,3 & 7,6 & 2,4 & & 6,5 & $<0,01$ & \\
expectativas & & & & & & & & \\
Interpersonal & 9,4 & 3,3 & 9,1 & 3,5 & & 0,7 & 0,48 \\
\hline
\end{tabular}

Fuente: Elaboración propia, a partir de los datos de estudio, 2016.

\section{Competencia para el cuidado en el hogar}

La competencia de cuidado de los cuidadores se analizó de manera total y por dimensiones.

Los dos grupos reportaron una competencia media, siendo ligeramente menor la de los cuidadores de adultos con cáncer. Se observa una diferencia estadísticamente significativa en la competencia total, siendo mayor la competencia en los cuidadores de niños. De igual forma se observa una diferencia en las dimensiones instrumental, disfrutar y anticipación, siendo mayor la competencia en los cuidadores de niños con cáncer (Tabla 4). 
Tabla 5. Competencia para el cuidador niño con cáncer-cuidador adulto con cáncer.

\begin{tabular}{l|cc|cc|c|c}
\hline \multicolumn{1}{c|}{ Variable } & \multicolumn{2}{|c|}{$\begin{array}{c}\text { Cuidador niño con } \\
\text { cáncer }\end{array}$} & \multicolumn{2}{c|}{$\begin{array}{c}\text { Cuidador adulto } \\
\text { con cáncer }\end{array}$} & t & p \\
& Media & DS & Media & DS & & \\
\hline $\begin{array}{l}\text { Total competencia } \\
\text { (0 a 180) }\end{array}$ & 155,9 & 19,8 & 149,4 & 19,9 & 2.06 & $<0.05$ \\
$\begin{array}{l}\text { Conocimiento (0 a 27) } \\
\text { Unicidad (0 a 39) }\end{array}$ & 24,5 & 4,1 & 23,6 & 5,7 & 1,1 & 0,25 \\
Instrumental (0 a 24) & 30,2 & 3,8 & 30,3 & 4,6 & 0,2 & 0,8 \\
Disfrutar (0 a 36) & 32,2 & 3,8 & 19,8 & 3,8 & 2,3 & $<0.05$ \\
Anticipación (0 a 18) & 16,3 & 6,3 & 28,4 & 5,7 & 4,69 & $<0.01$ \\
$\begin{array}{l}\text { Relación social e } \\
\text { interacción (0 a 36) }\end{array}$ & 31,0 & 4,9 & 15,1 & 2,8 & 2,74 & $<0.01$ \\
\hline
\end{tabular}

Fuente: Elaboración propia a partir de los datos de estudio, 2016.

\section{Discusión de resultados}

Como en otros estudios predominan mujeres en edades productivas asumiendo el rol de cuidador $(15,16,17)$ y para el caso de los niños con cáncer cuidadoras muy jóvenes, con unión marital y nivel socioeconómico bajo (18). Es alarmante la alta proporción de cuidadores sin vinculación laboral activa, con las consecuencias sobre gastos y restricciones asociadas a la condición económica. Se refleja también nivel de escolaridad bajo con limitado acceso a estudios universitarios. Podrían asociarse estos hallazgos con las instituciones donde fueron captados los participantes, entidades en donde predomina la atención de usuarios de régimen subsidiado de salud.

En cuanto a la sobrecarga para el cuidado, es evidente la diferencia entre la carga del cuidador del niño respecto a la del adulto, situación que puede estar relacionada por variables como que la mayoría son cuidadores únicos, con una dedicación diaria al cuidado de 22,2 horas, con alta permanencia en las instituciones hospitalarias para recibir los tratamientos oncológicos requeridos por los niños. $\mathrm{Al}$ igual que los hallazgos de Carreño y Arias (6), es posible que estos factores bien manejados sean susceptibles de abordar para incrementar la competencia de cuidado en el hogar, modulados por los apoyos con los que se cuente.

Los cuidadores tanto de niños como de adultos perciben altos niveles de apoyo familiar, social y religioso, componentes que pueden ser amortiguadores para el proceso de afrontamiento, la toma de decisiones, el manejo de problemas y la adquisición de competencias para el cuidado. Es de resaltar que los niveles de bienestar percibido de los cuidadores de niños con cán- 
cer son menores a los de los cuidadores de adultos, situación que puede estar relacionada con el impacto que representa asumir el nuevo rol de cuidar al niño enfermo versus la expectativa de tener a cargo un niño sano; las limitaciones en el desarrollo del rol como mujeres jóvenes productivas laboralmente, la tensión conyugal $(19,20)$, el desarrollo de roles simultáneos, entre otros factores (21).

La competencia para el cuidado en el hogar de forma global y en cada una de sus dimensiones está dentro de rangos medios, hallazgos que coinciden con estudios realizados en cuidadores familiares de personas con enfermedades crónicas en Colombia $(7,8,12)$. Es de señalar que los pacientes y sus cuidadores reciben instrucciones generales previas al inicio del tratamiento oncológico en las instituciones donde se realizó el estudio, factor que puede incidir en los resultados sobre las dimensiones conocimiento, instrumental y anticipación. Sin embargo, se reitera la pertinencia de fortalecer programas de seguimiento y continuidad que garanticen el conocimiento del cuidador respecto a la enfermedad, las terapéuticas farmacológicas y no farmacológicas, las indicaciones especiales, el manejo de dispositivos y equipos de la persona con cáncer, así como el conocimiento de las actividades a realizar propias de la cotidianidad tales como baño, vestido, alimentación, deambulación, entre otras.

Por otro lado, los componentes de afrontamiento, el bienestar que se tiene para comprender cómo se asume la responsabilidad del cuidado en casa y el fortalecimiento de las redes de apoyo primarias y secundarias con las que se cuenta $\mathrm{y}$ son factores que favorecen la labor de cuidado y se constituyen en retos para el sistema de salud dentro de la generación de esquemas de seguimiento del cuidado en el domicilio.

La diferencia entre los cuidadores familiares de niños y de adultos con cáncer permite ver que los cuidadores de niños tienen mayor nivel de competencia total y en las dimensiones instrumental, disfrutar y anticipación. Estos hallazgos podrían explicarse de varias formas: el tiempo y dedicación del cuidado se pueden relacionar con un aprendizaje en aspectos instrumentales, de recrearse y distraerse con las tareas propias del cuidado o con el respaldo propio de la edad, y con el desarrollo de mayor capacidad de planificar, identificar signos de alarma para consultar que tiende a ser mayor para los cuidadores de niños.

El menor tiempo de cuidado a partir del diagnóstico, el vínculo de parentesco siendo la mayor parte madres del niño enfermo, y las diferencias en la educación básica, podrían explicar también los mayores niveles de competencia en los cuidadores de niños (22).

El estudio tiene varias limitaciones. Se llevó a cabo en dos centros de atención en cáncer, reflejando variables propias de estos escenarios, cuidadores con escolaridad baja e ingresos económicos limitados. Hubiese sido interesante determinar el tipo, fuente y alcance de la capacitación e instrucción dada desde el momento del diagnóstico previo a la aplicación de los instrumentos, ya que sus percepciones y necesidades cambian a medida que reciben información sobre el manejo de la condición de salud.

Futuras investigaciones deben orientarse a determinar el impacto de intervenciones dirigidas a los cuidadores de niños 
y de adultos con cáncer para lograr una competencia óptima de cuidado en el hogar. La carga de cuidado es una variable a ser explorada, mediante abordajes cualitativos para determinar otras dimensiones que pueden estar relacionadas con los procesos y significados que se atribuyen a esta condición de salud.

\section{Conclusiones}

La sobrecarga para el cuidado de los cuidadores familiares de niños con cáncer es mayor a la de los cuidadores familiares de adultos con cáncer. Ahora bien, la competencia para el cuidado en el hogar

\section{Referencias}

1. Lindsey A, Bray F, Siegel R, Ferlay J, Lortet J, Jemal A. Global cancer statistics, 2012. CA Cancer J Clin. 2015; 65:87-108. doi: 10.3322/caac.21262

2. Baker E, Wellman N. Nutrition concerns in discharge planning for older adults: A need for multidisciplinary collaboration. J. Am. DietAssoc. 2005;105 (4):603-607.

3. Barrera L, Carrillo G, Chaparro L, Sánchez B. Modelo para abordar la carga del cuidado de la enfermedad crónica en Colombia. (Orinoquía) 2015;19 (1):89-99.

4. Galvis R, Pinzón L, Romero E. Comparación entre la habilidad de cuidado de cuidadores de personas en situación de enfermedad crónica en Villavicencio, Meta. Avances en Enfermería 2004; 22 (1): 4-26.

5. Safarabadi A, Maarefvand M, Biglarian $A$, Khubchandani J. Effectiveness es más alta en los cuidadores de niños que en los cuidadores de adultos de forma global y en las dimensiones de conocimiento, instrumental y anticipación. Se requiere focalizar aún más el apoyo a cuidadores familiares, según el receptor de cuidado. Programas para fortalecer la competencia de cuidado en el hogar y disminuir la carga son necesarios para atender y responder a la necesidad de los cuidadores familiares de personas con cáncer. Es necesario formar al recurso humano de enfermería para que implementen programas de fortalecimiento de la competencia para cuidar en el hogar, por ser este un fenómeno y dominio de la disciplina de enfermería.

of a Brief Psychosocial Intervention on Quality of Life of Primary Caregivers of Iranian Children with Cancer: A Randomized Controlled Trial. Journal Pediatr Nurs. 2016;31(4): 262-270. doi: 10.1016/j.pedn. 2016.01.002

6. Carreño S, Arias M. Competencia para cuidar en el hogar y sobrecarga en el cuidador del niño con cáncer. Gaceta Mexicana de Oncología. 2016;15(6):336343. Doi: https://doi.org/10.1016/j.gamo. 2016.09.009

7. Sánchez L, Carrillo G. Competencia para el cuidado en el hogar díada persona con cáncer en quimioterapia. Cuidador familiar. Psicooncología. 2017;14(1): 137-148.

8. Achury DM, Restrepo A, Torres NM, Buitrago AL, Neira NX, Devia P. Competencia de los cuidadores familiares para cuidar a los pacientes con falla cardíaca. Rev Cuid. 2017; 8(3):1721-1732.

9. Peñaranda EH, Rico YA, Rozo YA, Vega OM. Cuidadores familiares: competencia 
en la atención de enfermos crónicos. Rev. cienc. cuidad. 2018;15(1): 30-41.

10. Carrillo G, Barreto R, Arboleda L, Gutiérrez O, Melo B, Ortiz T. Competencia para cuidar en el hogar de personas con enfermedad crónica y sus cuidadores en Colombia. Rev Fac Med. 2015;63:665675.

11. Carrillo G, Sánchez Herrera B, Arias EM. Validación de un instrumento para la evaluación de la competencia de cuidado en el hogar del cuidado familiar de una persona con enfermedad crónica. Investigación y educación en enfermería. 2015;33(3):449-455. http://dx.doi. org/10.17533/udea.iee.v33n3a08

12. Breinbauer $H$, Vásquez $H$, Mayanz $S$, Guerra C, Millán T. Validación en Chile de la Escala de Sobrecarga del Cuidador de Zarit en sus versiones original y abreviada. Revista Med. Chile. 2009;137(1): 657665.

13. Barrera R, Campos ME, Carrillo GM, Coral R, Chaparro L, Durán M, et al. Entrevista de percepción de carga del cuidado de Zarit: pruebas psicométricas para Colombia. Aquichan. 2015;15(3): 368-380.

14. Resolución 008430 de 1993: Por la cual se establecen las normas científicas, técnicas y administrativas para la investigación en salud. República de Colombia:Ministerio de Salud; 4 de octubre de 1993.

15. Krikorian A, Vélez C, González O, Palacio C, Vargas J. La experiencia de sufrimiento en cuidadores principales de pacientes con dolor oncológico y no onco- lógico. Preav. Enferm. 2010. 28(1):13-20. 16. Puerto H. Soporte social percibido en los cuidadores familiares de personas en tratamiento contra el cáncer. Cuidarte. 2017; 8(1): 1407-1422.

17. Ávila J, Vergara M. Calidad de vida en cuidadores informales de personas con enfermedades crónicas. Aquichan. 2014;14(3): 417-429.

18. Pöder U, Ljungman G, Von Essen L. Parents' perceptions of their children's cancer-related symptoms during treatment: A prospective, longitudinal study. J Pain Symptom Manage. 2010;40:661670.

19. Rubira do Espirito Santo EA, Munhoz Gaíva MA, Martinez Espinosa M, Barbosa DA, Silva AG. Taking care of children with cancer: Evaluation of the caregivers' burden and quality of life. Rev Latatino-Am Enfermagem. 2011;19:515-522.

20. Litzelman K, Catrine K, Gangnon R, Witt P. Quality of life among parents of children with cancer or brain tumors: The impact of child characteristics and parental psychosocial factors. Qual Life Res. 2011;20(8):1261-1269. Doi: 10.1007/ s11136-011-9854-2.

21. Carrillo G, Barrera L, Sánchez B, Carreño $S$, Chaparro $L$. Efecto del programa de habilidad de cuidado para cuidadores familiares de niños con cáncer. Rev Colomb Cancerol. 2014; 18(1):18-26.

22. Carrillo G, Sánchez B, Barrera L. Habilidad de cuidado de cuidadores familiares de niños y adultos con enfermedad crónica. Index Enferm. 2014; 23(3): 129-133. 
